\title{
Ciclo diario y anual de variables troposféricas y oceánicas en la Isla del Coco, Costa Rica
}

\author{
Eric J. Alfaro \\ Centro de Investigación en Ciencias del Mar y Limnología (CIMAR), Centro de Investigaciones Geofísicas (CIGEFI) \\ y Escuela de Física, Universidad de Costa Rica. Ciudad Universitaria Rodrigo Facio, 11501-2060 San José, Costa \\ Rica; erick.alfaro@ucr.ac.cr
}

Recibido 14-IX-2007. C Corregido 02-V-2008. Aceptado 11-VI-2008.

\begin{abstract}
Diel and annual cycle of tropospheric and oceanic variables in Cocos Island, Costa Rica. Meteorological records from gauge stations at Isla del Coco (Cocos Island), Costa Rica, were analyzed. These data are from an automatic weather station and from two pluviograph stations, covering the period 1997-2005. Time series from other historical records and from the nearest island grid point from different public geophysical data sets were also used. The focus was on rainfall, wind, radiation, relative humidity, and air and sea temperature series. Daily and annual cycles were calculated for all the records with hourly resolution and their intraseasonal features were studied, such as maxima values, minima values and transitional periods. Comparisons were done between the stations records and the grid (with broader spatial resolution data). In general, seasonal variations at Isla del Coco were observed to be mainly associated with the meridian migration of the Inter Tropical Convergence Zone; the island is under its direct influence from boreal spring to autumn. Rev. Biol. Trop. 56 (Suppl. 2): 19-29. Epub 2008 August 29.
\end{abstract}

Key words: Isla del Coco, Cocos Island, eastern tropical Pacific, climatology, intra-seasonal variability, Inter Tropical Convergence Zone.

Las mediciones de variables atmosféricas y oceanográficas en la Isla del Coco, Costa Rica, han sido dispersas, tanto en tiempo como en espacio. El principal factor que ha contribuido a eso ha sido la dificultad del desplazamiento hasta el lugar y su costo económico elevado ya que el punto continental más cercano es Cabo Blanco, en la Península de Nicoya, Costa Rica a 495 km (Lizano 2001), por lo que tales observaciones eran hechas en expediciones de oportunidad (Pittier 1898, Rojas-Acuña 1964, Cortés 2008). Por ejemplo, Protti (1964) hizo una de las primeras recopilaciones de observaciones y datos ocasionales y clasificó el clima de la isla semejante al tipo tropical lluvioso con una temperatura media de $25^{\circ} \mathrm{C}$, sin una época seca definida y con vientos dominantes del Suroeste.
Sin embargo, estas observaciones no permitían su uso como una serie de tiempo. Uno de los primeros esfuerzos para tomar observaciones sistemáticas en esta isla lo realizó el Instituto Meteorológico Nacional (IMN) de Costa Rica con el emplazamiento de un pluviómetro en febrero de 1979 (Fernández 1984). Posteriormente, el IMN trabajó en forma conjunta con el Centro de Investigación Científica y de Educación Superior de Ensenada (CICESE) en México y operaron una estación semi-automática en la isla a partir de mayo de 1979 por espacio de aproximadamente dos años. Estos datos fueron analizados preliminarmente por Reyes y Vogel (1981), y corregidos y complementados posteriormente por Fernández (1984), siendo estos unos de los primeros análisis de series de tiempo tomadas in situ en dicho lugar. 
Posteriormente, el acceso y ordenamiento de las bases de datos meteorológicos y oceanográficos recolectados globalmente, el uso de sensores remotos en las últimas décadas y los diferentes estudios de re-análisis usando modelos numéricos (e.g. da Silva et al. 1994, Xie y Arkin 1996, Kaplan et al. 1998, Magaña et al. 1999), ha permitido la elaboración de series de tiempo lo suficientemente extensas para su uso en estudios climáticos recientes en la región del Pacífico Tropical Este (Amador et al. 2006).

Sin embargo, esto último más bien ha incrementado la necesidad de contar con registros de datos geofísicos in situ en la isla principalmente por dos razones. La primera es que ellos permiten validar los datos obtenidos por medio de otras metodologías, e.g. sensores remotos, y la segunda es que estos son útiles a la hora de realizar análisis de ajuste de escala para estudios de impactos locales, principalmente para trabajos relacionados con el cambio y la variabilidad climática (IPCC 2007), debido a que los datos obtenidos con otras técnicas metodológicas son por lo general de una escala espacial y temporal mayor a la que se puede registrar por medio de una estación meteorológica emplazada en el lugar. Cabe destacar que desde el año 2005 no se están realizando observaciones meteorológicas de ningún tipo en forma sistemática en la Isla del Coco, por lo tanto el registro de las series de tiempo se ha interrumpido y no se tiene conocimiento de cuándo podría volver a operar una estación meteorológica en esta isla.

El objetivo de este trabajo es el de presentar algunos aspectos climáticos de la Isla del Coco, para lo que se analizó el ciclo diario y anual de ciertas variables troposféricas y oceánicas en la Isla del Coco, utilizando los registros meteorológicos de las estaciones que han funcionado en la isla. También se plantea su comparación con estudios anteriores y con datos de bases globales públicas que cubran en su dominio a la isla.

\section{MATERIALES Y MÉTODOS}

Se recolectaron los registros meteorológicos de las estaciones que han funcionado recientemente en la Isla del Coco, Costa Rica, y se realizó el respectivo control de calidad de los datos. Se recopilaron los datos horarios de una estación meteorológica automática del IMN $\left(05^{\circ} 33^{\prime} \mathrm{N} 87^{\circ} 03^{\prime} \mathrm{W}\right.$, código 200002, del 30/08/1998 al 01/11/2002) y datos de dos pluviómetros, uno del IMN (0532'46”'N 8702'42”'W, código 200001, del 01/05/1997 al 17/11/2004) con datos horarios y otro del Instituto Costarricense de Electricidad (ICE) $\left(05^{\circ} 33^{\prime} \mathrm{N} 87^{\circ} 03^{\prime} \mathrm{W}\right.$, código 100002 , del 22/06/2002 al 07/07/2005) con datos diarios.

Se hizo énfasis en la recolección de registros de precipitación, viento, radiación, humedad relativa y la temperatura superficial del aire. Para la serie de datos de la estación meteorológica automática, se notó que los registros de humedad relativa mostraban un salto a partir de las 11 horas del 29/08/2001 y los datos de viento y radiación mostraron un salto a partir de las 12 horas del 27/04/2002. Esos fueron visibles tanto en sus valores promedio como en su varianza. Los registros posteriores a los saltos fueron normalizados y corregidos luego con el promedio y la desviación estándar de los registros previos a los mismos. Debido a la falta de metadatos no se pudo determinar la causa de estas anomalías, así como también la explicación de la ausencia de algunos datos en los registros.

También se obtuvieron las series de tiempo de los puntos de rejilla más cercanos a la isla de las bases de datos de precipitación y de temperatura compilados de estaciones medidoras en superficie, estimados de percepción remota y de modelos numéricos, por la Universidad Nacional Autónoma de México, UNAM (Magaña et al. 1999), del 01/1958 al 12/1998 (datos centrados en $5^{\circ} 30^{\prime} \mathrm{N} 87^{\circ} 00^{\prime} \mathrm{W}$, de una rejilla de $0.5^{\circ} \times 0.5^{\circ}$ ), de precipitación de Xie y Arkin (1996) del 01/1979 al 02/1999 (datos centrados en $6^{\circ} 15^{\prime} \mathrm{N} 86^{\circ} 15^{\prime} \mathrm{W}$, de una rejilla de $2.5^{\circ} \times 2.5^{\circ}$ ), y de la Temperatura Superficial del Mar (TSM) de da Silva et al. (1994) del 01/1945 al 06/2000 (datos centrados en $5^{\circ} 30^{\prime} \mathrm{N} 87^{\circ} 30^{\prime} \mathrm{W}$ de una rejilla de $1^{\circ} \mathrm{x} 1^{\circ}$ ]) y de Kaplan et al. (1998) del 01/1949 al 08/2000 (datos centrados en $5^{\circ} \mathrm{N} 87^{\circ} \mathrm{W}$, de una rejilla 
de $2^{\circ} \times 2^{\circ}$ ). Adicionalmente, se obtuvieron los registros históricos descritos en Reyes y Vogel (1981) y Fernández (1984).

Para todas las variables con registros de datos horarios de la estación automática, se obtuvieron sus ciclos diarios y anuales. Lo anterior se realizó por medio del cálculo del promedio aritmético de todos los datos recolectados para un mes y una hora determinada. Se estudió posteriormente sus características intraestacionales, tales como máximos, mínimos y épocas de transición.

También se utilizaron los datos del viento en superficie del escaterómetro QuikSCAT sobre la Isla del Coco (datos centrados en $5.75^{\circ} \mathrm{N} 87.25^{\circ} \mathrm{W}$, de una rejilla de $0.5^{\circ} \times 0.5^{\circ}$ ) entre setiembre 1999 y mayo 2006 y los datos de los globos piloto del proyecto PAC-SONET, lanzados desde la Isla del Coco entre mayo 1997 y enero 1999 (Douglas et al. 1999).

\section{RESULTADOS}

Precipitación: El periodo de mayor precipitación se ubica entre mayo y octubre, principalmente en horas de la tarde (Fig. 1a, ver también Fig. 2a). El primer trimestre del año se caracterizó por ser el periodo con mayor temperatura superficial del aire (Fig. 1b), mayor radiación total (Fig. 1c), menor humedad relativa (Fig. 1d) y menor velocidad del viento (Fig. 1e, f), principalmente en horas cercanas al medio día. Los ciclos anuales de precipitación y temperatura calculados por Fernández (1984) coinciden en términos generales con los presentados aquí (Fig. 1).

Se observaron valores pequeños en el flujo de radiación total. Esto concuerda con los valores altos obtenidos por Amador et al. (2006) para el promedio anual de la cobertura nubosa sobre la isla, la cual es de aproximadamente 0.7-0.75 décimas. La razón de lo anterior son los procesos de convección profunda que se desarrollan en la región asociados a la Zona de Convergencia Inter-Tropical (ZCIT). Ellos también encontraron valores del flujo de radiación saliente de onda larga de aproximadamente $44-46 \mathrm{Wm}^{-2}$ y del flujo de calor latente de alrededor de 60-80 $\mathrm{Wm}^{-2}$. Estas cantidades son mantenidas a lo largo del año por los valores altos observados en esta región del flujo de radiación incidente de onda corta de aproximadamente $190 \mathrm{Wm}^{-2}$, siendo esto típico en las regiones ecuatoriales. Según estos autores, lo anterior explica que el flujo de energía neto a nivel superficial sea muy pequeño en las regiones circundantes a la isla, ya que mucha de la energía que alcanza el océano es luego cedido a la atmósfera suprayacente en forma de calor latente o trasportado por las corrientes oceánicas (ver también Lizano 2008).

Vientos: El ciclo diario del viento tiene tres periodos distintos a lo largo del año (Fig. 1e). En el primero, entre diciembre y abril, la magnitud del viento es menor durante el día, principalmente en horas de la tarde, comparado con las horas de la noche o temprano en la mañana. $\mathrm{Al}$ ser esta la época del año en la cual la influencia del flujo sinóptico del suroeste es menor, lo anterior podría reflejar que los altos valores de temperatura del aire diurnos durante esta época reducen el gradiente de temperatura mar-tierra, sin embargo son necesarios estudios posteriores con series de tiempo mayores y medidas in situ, para validar tal hipótesis y determinar si esta es la causa de tal reducción. El segundo periodo, entre mayo y agosto, la magnitud del viento es muy similar a lo largo de todo el día y en el tercer periodo, de setiembre a noviembre, la magnitud del viento es mayor durante las horas del día que durante las de la noche. Estos dos últimos periodos están de acuerdo con el ciclo diario presentado por Fernández (1984), cuyo ciclo anual, con un valor promedio anual de 2.9 $\mathrm{ms}^{-1}$, concuerda en términos generales con el obtenido en este trabajo. Las variaciones estacionales del ciclo diario del viento podrían estar explicadas por la predominancia de sistemas mesoescalares durante ciertas épocas del año o por influencias orográficas, sin embargo las series de tiempo con que se cuenta actualmente no permiten su determinación, por lo que la toma de más datos in situ y la realización de más estudios de modelado ayudarían al entendimiento de este ciclo. 
a)

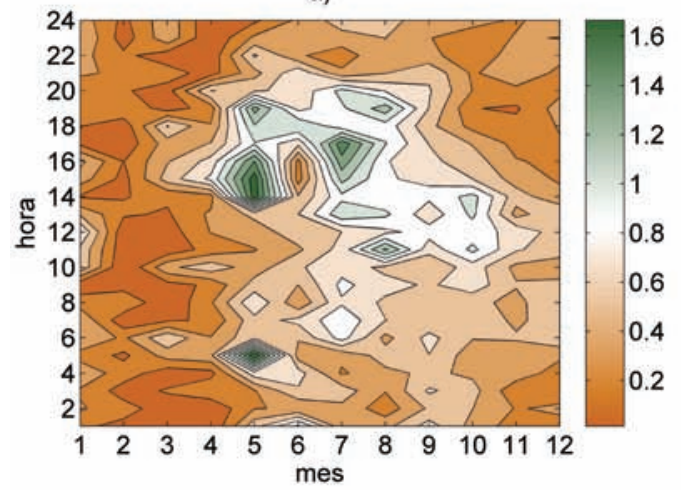

c)

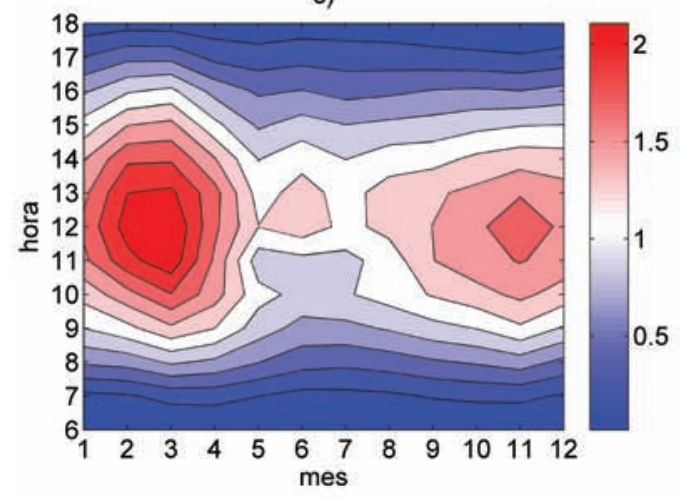

e)

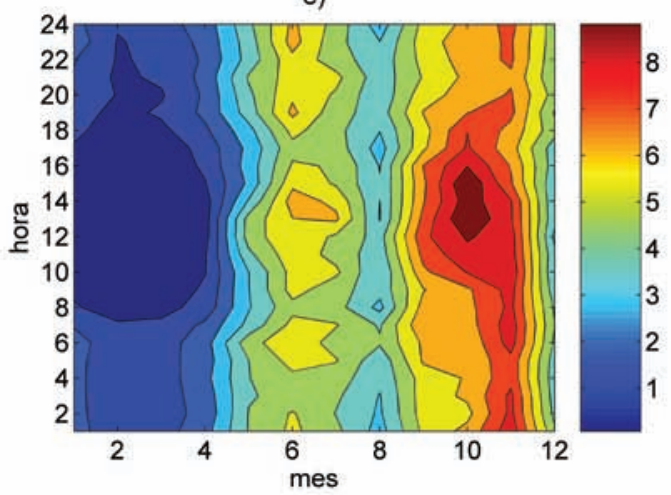

b)

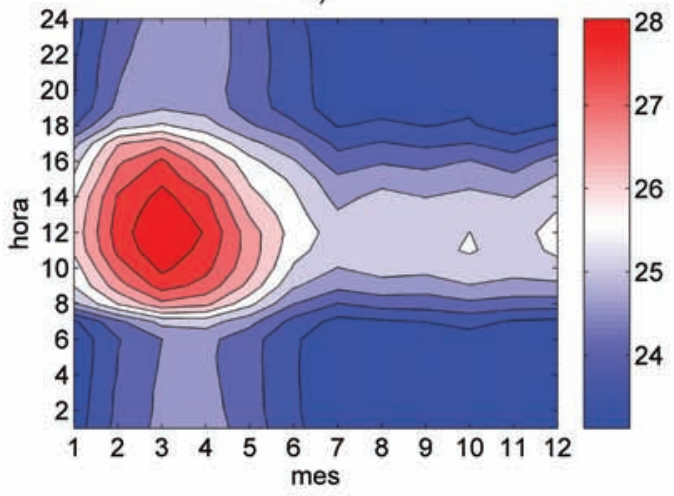

d)

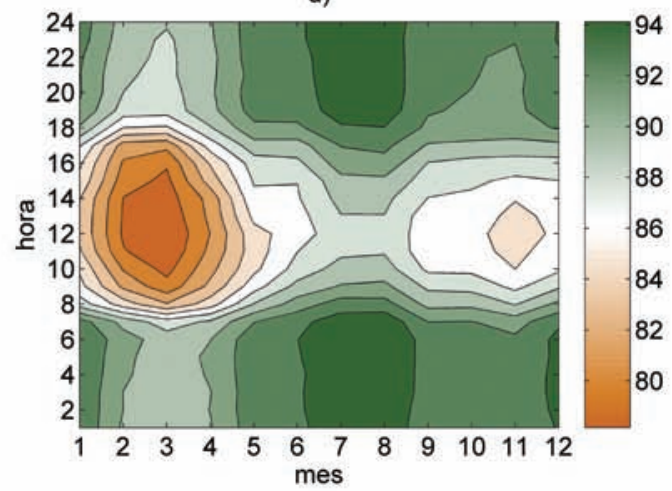

f)

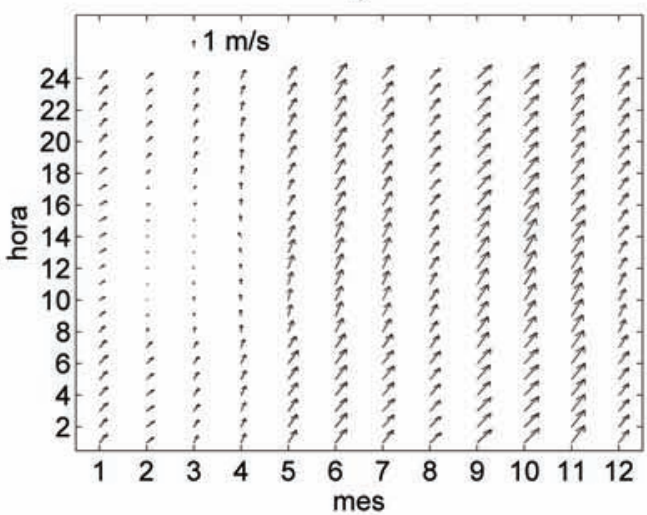

Fig. 1. Ciclo anual (eje x) y diario (eje y) de los datos de la estación automática de la Isla del Coco para a) precipitación $(\mathrm{mm}), \mathrm{b})$ temperatura superficial del aire $\left.\left({ }^{\circ} \mathrm{C}\right), \mathrm{c}\right)$ radiación $\left.\left(\mathrm{MJm}^{-2}\right), \mathrm{d}\right)$ humedad relativa (\%), e) el cuadrado de la rapidez del viento $\left(\mathrm{m}^{2} \mathrm{~s}^{-2}\right)$ y f) el vector del viento $\left(\mathrm{ms}^{-1}\right)$.

Fig. 1. Annual (x axis) and daily (y axis) cycle of the automatic weather station data at Isla del Coco for a) precipitation $(\mathrm{mm}), \mathrm{b})$ air surface temperature $\left.\left({ }^{\circ} \mathrm{C}\right), \mathrm{c}\right)$ radiation $\left.\left(\mathrm{MJm}^{-2}\right), \mathrm{d}\right)$ relative humidity $(\%)$, e) the square of the wind velocity $\left(\mathrm{m}^{2} \mathrm{~s}^{-2}\right)$ and $\left.\mathrm{f}\right)$ the wind vector $\left(\mathrm{ms}^{-1}\right)$. 
a)

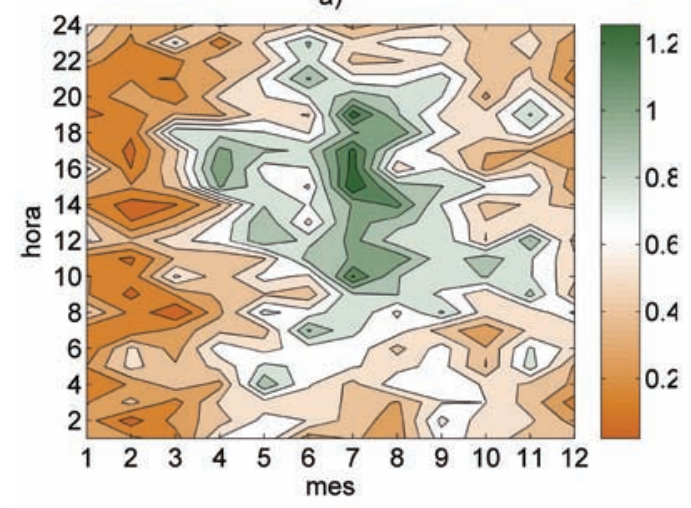

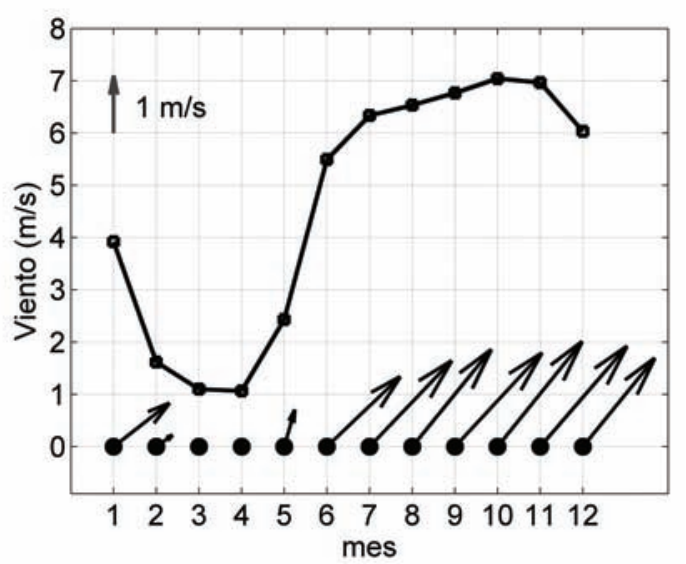

Fig. 2. a) Ciclo anual (eje x) y diario (eje y) de los datos del pluviómetro del IMN en la Isla del Coco (mm), y b) ciclo anual del vector y la magnitud del viento utilizando los datos del escaterómetro QuikSCAT sobre la Isla del Coco (ms $\left.{ }^{-1}\right)$.

Fig. 2. a) Annual (x axis) and daily (y axis) cycle of the IMN (National Weather Service, Costa Rica) rain gauge data at Isla del Coco (mm), and b) annual cycle of the wind vector and its magnitude using the QuikSCAT scatterometer data over Isla del Coco $\left(\mathrm{ms}^{-1}\right)$.

La dirección predominante del viento fue del suroeste y el periodo de mayor energía cinética fue el bimestre octubre-noviembre (Figs. 1e, f). Resultados similares se obtuvieron con los datos del viento del escaterómetro QuikSCAT sobre la Isla del Coco (Fig. 2b) y con los de los globos piloto del proyecto PACSONET (Douglas et al. 1999), lanzados desde la Isla del Coco entre mayo de 1997 y enero de 1999 (Fig. 3). También están de acuerdo con los valores obtenidos por Amador et al. (2006), haciendo uso de los datos del re-análisis del NCEP/NCAR. Estos autores además encontraron un valor promedio anual de la componente meridional del viento de entre $4-5 \mathrm{~ms}^{-1}$, ubicándose la isla en una región con un esfuerzo superficial del viento ciclónico de aproximadamente 3-6 x 10-8 $\mathrm{Nm}^{-3}$. Lo anterior ubica a la isla en una zona de convergencia importante de los vientos alisios. Valores similares, tanto en dirección como en magnitud, para el esfuerzo superficial del viento, fueron encontrados por Chelton et al. (2004), Kessler (2006) y Willet et al. (2006). Estos autores agregan, al analizar el rotor del viento, que la Isla del Coco se encuentra en una zona de transición meridional con rotación anticiclónica, asociada al hundimiento de aguas al sur de la isla y rotación ciclónica, asociada al afloramiento de aguas al norte de la isla.

Lizano (2008) explica que lo observado en el esfuerzo del viento sobre la superficie del océano ayuda a entender la dirección

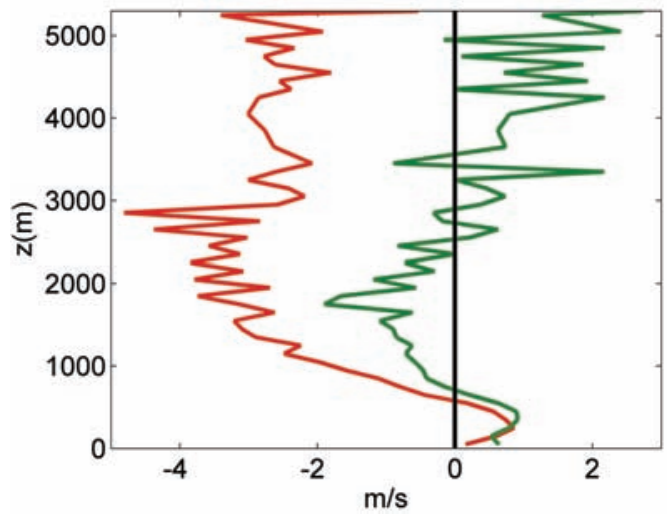

Fig. 3. Componente zonal promedio u (línea roja), y meridional v (línea verde), de los globos piloto del proyecto PAC-SONET, lanzados desde la Isla del Coco entre mayo 1997 y enero 1999.

Fig. 3. Zonal component u (red line) and meridian component $\mathrm{v}$ (green line) average for the pilot balloons of the PAC-SONET project, launched from Isla del Coco between May 1997 and January 1999. 
predominante del oleaje en los alrededores de la Isla del Coco, el cual proviene del sur-suroeste entre abril y noviembre y del oeste-suroeste entre diciembre y marzo.

Los vientos superficiales del suroeste, producto del cambio de dirección de los alisios del anticiclón del Pacífico Sur por efecto de Coriolis (Muñoz et al. 2002, Amador et al. 2006, Lizano 2008), están confinados a una capa de menos de mil metros de altitud ya que arriba de ellos la dirección predominante del viento es del noreste hasta aproximadamente los $2500 \mathrm{~m}$, arriba de esta capa y hasta los $5000 \mathrm{~m}$ de altitud, los vientos predominantes se observaron del sureste (Fig. 3). Zhang et al. (2004) y Amador et al. (2006) describen la existencia de una circulación atmosférica meridional somera sobre el Pacífico Ecuatorial Este. Basados en varios conjuntos de datos independientes, estos autores encontraron un flujo de retorno hacia el ecuador inmediatamente arriba de la capa límite atmosférica, lo cuál se contrapone con el concepto clásico de una única circulación profunda Sur-Norte tipo celda de Hadley, cuyo flujo hacia el norte se ubica en la troposfera alta.

Comparaciones - precipitación: Los ciclos anuales de registros de precipitación de las estaciones meteorológicas y su comparación con los registros de las bases de datos con mayor escala espacial se presentan en la Fig. 4. Entre el 72 y el 75\% de la precipitación acumulada anualmente se registra entre abril y octubre (Fig. 4a). Los valores promedio para los acumulados anuales fueron de 5137.8, 5206.4 y $4758.9 \mathrm{~mm}$, para los pluviógrafos del IMN, del ICE y la estación automática del IMN, respectivamente, con ámbitos dados por $\pm \sigma$ de 7363.4-2912.2, 6458.6-3954.3 y 6533.9-2983.9. Estos valores de precipitación acumulada anual son similares a lo reportado por Fernández (1984) para el pluviómetro, $6097.4 \mathrm{~mm}$, pero no para la estación semiautomática, $3125.8 \mathrm{~mm}$. Además, de abril a octubre, entre el 84 y el $87 \%$ de los días registran precipitación en un mes determinado (Fig. 4b) y fueron los meses en donde se ubicaron los eventos máximos de precipitación acumulada en un solo día (Fig. 4c). En todos los casos de la Fig. 4a mínima precipitación fue febrero, con valores acumulados entre los 100 y los 200 mm y en el cual entre el 40 y el 50\% de los días presentan precipitación (los registros presentados en esta figura no muestran un consenso en cuanto al mes de máxima precipitación). Debido a lo anterior, se puede definir una temporada en donde las lluvias disminuyen entre noviembre y marzo, aunque esta no se podría calificar como una época "seca", ya que los valores anuales de la precipitación superan por mucho los valores de la evaporación (Fiedler y Talley 2006) que en la región es de aproximadamente 600-900 mm (Amador et al., 2006).

Al comparar los datos de las estaciones meteorológicas (Fig. 4a) con los de la Fig. 4d, correspondientes al conjunto de Xie \& Arkin (1996) y Magaña et al. (1999), se nota que en general estos últimos subestiman los acumulados mensuales. Los valores promedio para los acumulados anuales fueron de 3019.1 y 2031.4 mm, para los datos de Xie \& Arkin (1996) y Magaña et al. (1999), respectivamente. Esta subestimación fue encontrada también por Amador et al. (2006) al usar los datos elaborados por da Silva et al. (1994), con valores de acumulación anual de 3600 mm. En términos generales, la forma de la curva del ciclo anual de los datos de Xie y Arkin (1996), coincide mejor con los de las estaciones que aquellos de Magaña et al. (1999), los cuales presentan muy poca variabilidad a lo largo del año. Además, de abril a octubre, se acumula el 78 y el $60 \%$ de la precipitación, respectivamente en estos conjuntos de datos, lo cual contrasta con los registros de las estaciones de la isla (Fig. 4a).

Cabe destacar que las diferencias encontradas entre los ciclos presentados en las Figs. 1a, 2a y 4, así como con estudios previos (e.g. Reyes y Vogel 1981, Fernández 1984) para los registros de precipitación, se podrían explicar al considerar que la precipitación en general presenta una variación tanto espacial como temporal muy alta y los conjuntos de datos con los cuales se obtuvieron los resultados para elaborar estas figuras pertenecen a distintos 
a)

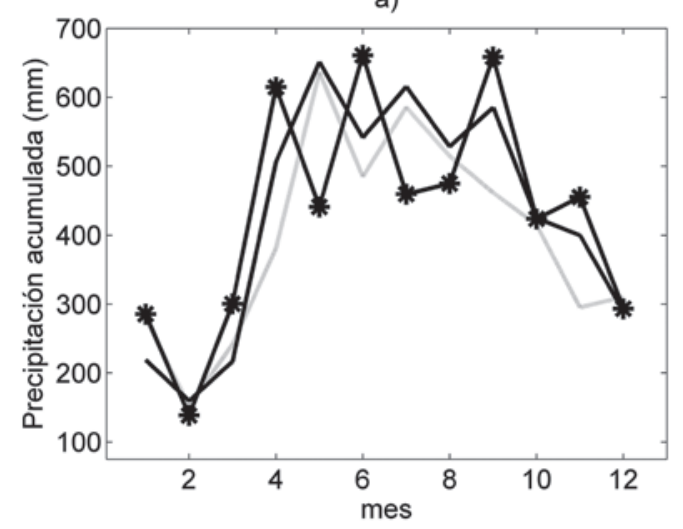

c)

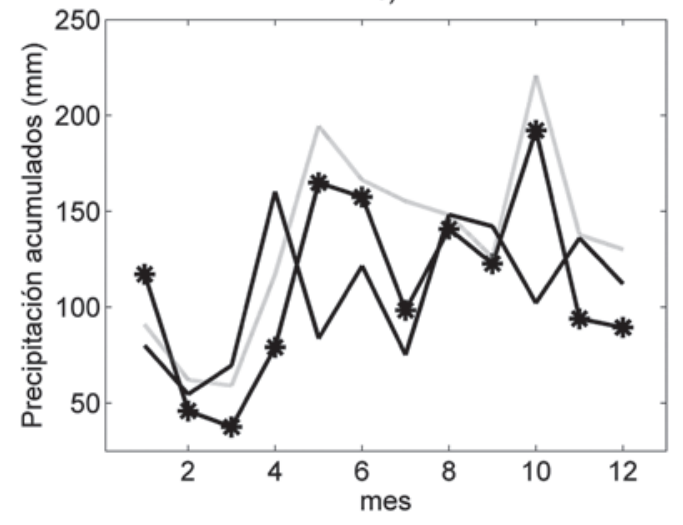

b)

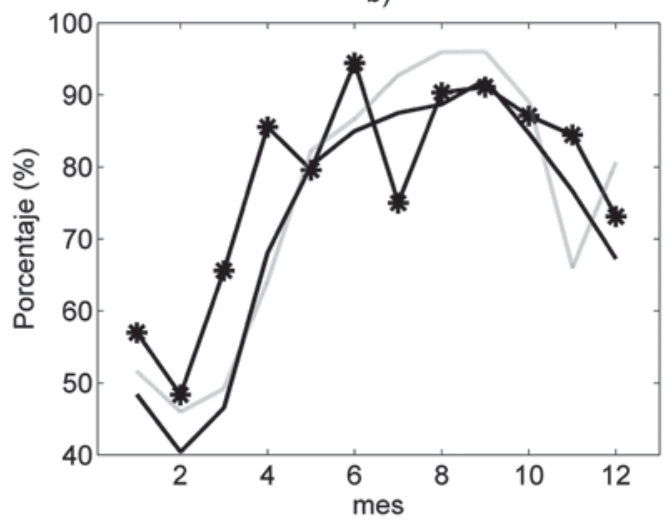

d)

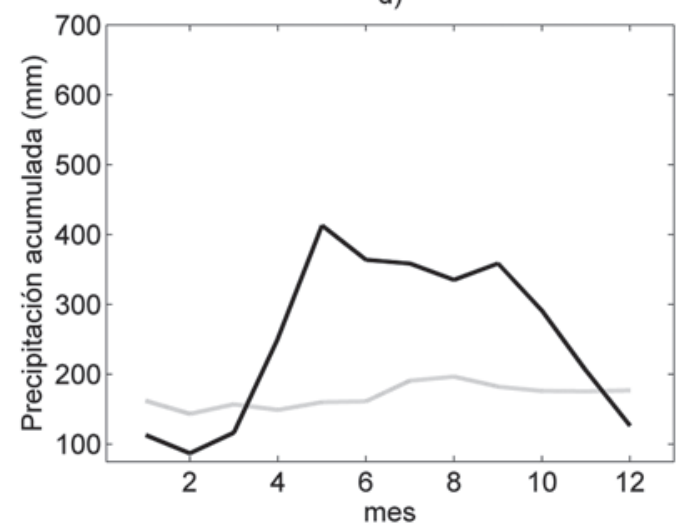

Fig. 4. Ciclo anual para a) el acumulado de precipitación mensual, b) el porcentaje de días con precipitación y c) los valores máximos acumulados durante un solo día en un mes determinado. Las líneas continuas corresponden a los datos del pluviómetro del IMN (1997-2002), las continuas con asteriscos a las del ICE (2002-2005) y las líneas grises a la estación automática del IMN (1998-2002). d) Ciclo anual para el acumulado de precipitación mensual de los datos elaborados por Magaña et al.(1999) línea gris y por Xie y Arkin (1996) línea continua.

Fig. 4. Annual cycle for a) monthly precipitation accumulates, b) percentage of days with precipitation and c) the maximum values accumulated during a day for a particular month. The continuous lines correspond to the rain gauge of the IMN (1997-2002), the continuous lines with asterisks to the rain gauge of the ICE (2002-2005) and the gray lines to the automatic weather station of the IMN (1998-2002). d) Annual cycle of the cummulative monthly rainfall from the data elaborated by Magaña et al.(1999) gray line and by Xie and Arkin (1996) continuous line.

sensores, periodos de tiempo dispares y además, de distinta escala espacial.

Comparaciones - temperatura: Una comparación de los registros mensuales de la temperatura superficial de aire en la estación automática del IMN con los compilados por la UNAM y por Magaña et al. (1999) y con los de la TSM obtenidos por da Silva et al. (1994) y de Kaplan et al. (1998) se presentan en la Figura 5. Para todos los registros de esa figura, el bimestre del año más cálido fue el de marzoabril y las menores temperaturas se registraron durante el segundo semestre (julio-diciembre). El valor anual promedio de la temperatura en la estación automática fue de $24.6^{\circ} \mathrm{C}$, lo que contrasta con el resto de los registros, en donde se observaron temperaturas anuales promedio 


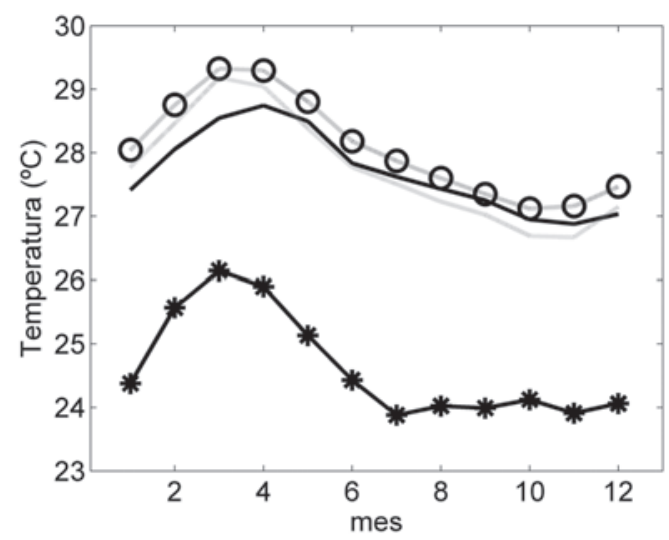

Fig. 5. Ciclo anual para la temperatura superficial del aire en la estación meteorológica automática, línea continua con asteriscos, los datos elaborados por la UNAM y descritos por Magaña et al. (1999) línea a trazos con círculos, y para la TSM de los datos elaborados por da Silva et al. (1994) y de Kaplan et al. (1998) líneas continua y gris, respectivamente.

Fig. 5. Annual cycle of the air surface temperature at the automatic weather station, continuous line with asterisks, the data generated by UNAM and described by Magaña et al. (1999) line with circles, and for the SST of the data elaborated by da Silva et al. (1994) and by Kaplan et al. (1998) continuous lines and gray line, respectively.

entre los 27.7 y $\operatorname{los} 28.1^{\circ} \mathrm{C}$. Esto podría evidenciar que en la reconstrucción hecha por Magaña et al. (1999), se le dio mayor peso a los valores de TSM. Por otro lado, la variación del ciclo anual de la temperatura, medida con la estación automática, coincide con lo reportado por Fernández (1984) para la temperatura del aire y del suelo, cuyos valores anuales promedio fueron de 23.6 y $25.5^{\circ} \mathrm{C}$, respectivamente. Los valores anuales promedio para la TSM y su variación están de acuerdo con lo presentado por Chelton et al. (2004), Fiedler y Talley (2006), Willet et al. (2006) y Lizano (2008), los cuales usaron datos obtenidos de diferentes fuentes tales como sensores remotos, batitermógrafos, CTD y botellas.

\section{DISCUSIÓN}

Las variaciones encontradas en este trabajo del ciclo anual de las diferentes variables concuerdan en general con los descritos por Amador et al. (2006), los cuales describen que sobre el Pacífico Tropical Este, entre el ecuador y los $10^{\circ} \mathrm{N}$, se encuentra un cinturón nuboso, asociado con la convergencia de los vientos alisios en latitudes bajas. Estos vientos transportan humedad y fuerzan el aire hacia una región de fuerte movimiento ascendente, la cual es llamada la Zona de Convergencia InterTropical (ZCIT) y es una zona de baja presión superficial atmosférica $\sim 989.6 \mathrm{hPa}$ como promedio anual en la Isla del Coco (Fernández 1984), fuerte actividad convectiva e intensa precipitación. Las variaciones estacionales observadas en la Isla del Coco se pueden explicar por la migración meridional de la ZCIT, la cual se ubica en su posición más al sur durante el invierno boreal (Amador et al. 2006), por lo que la isla se encuentra bajo la influencia de la ZCIT prácticamente desde la primavera hasta el otoño boreal (Alfaro y Amador 1997).

Por otro lado, las variaciones interanuales de las distintas variables descritas también podrían estar relacionadas con las variaciones interanuales de la ZCIT, cuya migración meridional es altamente dependiente del ENOS. Por ejemplo, según Philander (1990) en un ciclo estacional regular, la ZCIT comienza su traslación hacia el norte en abril o mayo, sin embargo durante un evento cálido del ENOS (El Niño), la ZCIT no inicia su viaje hacia el norte sino hasta mayo-junio, lo cual redundaría en una mayor precipitación asociada a una mayor temperatura superficial del mar y del aire en la Isla el Coco.

Por último, las diferencias encontradas en este estudio en los ciclos diarios y anuales de una misma variable, medida por diferentes métodos, obtenidas de diferentes conjuntos de datos o presentadas en otros estudios (ver Figs. 1a, 2a, 4, y Fernández 1984), hace que la necesidad planteada por Pittier a finales del siglo XIX (ver Anexo) cobre actualidad. Se deben realizar esfuerzos para realizar observaciones sistemáticas in situ de diferentes variables geofísicas en la Isla del Coco, que permitan la obtención de series de tiempo y ayuden al estudio del sistema climático. Lo anterior contribuye a un 
mayor entendimiento de los aspectos oceanográficos del Pacífico Tropical del Este, tanto físicos (e.g. Amador et al. 2006, Fielder y Talley 2006, Kessler 2006, Willet et al. 2006, Lizano 2008), como biológicos (e.g. Ballance et al. 2006, Fernández-Álamo y Färber-Lorda 2006, Pennintong et al. 2006, Morales-Ramírez 2008). Sin embargo, a la fecha esta toma de datos sistemática en la Isla del Coco no ha sido posible de realizar, debido a que todavía no se han podido solventar las dificultades logísticas y económicas que ello implicaría.

\section{AGRADECIMIENTOS}

A José Villegas y Tito Maldonado por la elaboración de la base de datos y de las figuras. Este trabajo se realizó gracias al apoyo de los siguientes proyectos de la Vicerrectoría de Investigación, Universidad de Costa Rica: 808A6-053, 808-A6-050, 808-A7-401, 805-98-506 y de la Vicerrectoría de Acción Social: ED-1977.

\section{RESUMEN}

Se recolectaron los registros meteorológicos de las estaciones que funcionaron en la Isla del Coco, Costa Rica, pertenecientes a una estación meteorológica automática y a dos pluviógrafos, para el periodo 1997 - 2005. También se obtuvieron las series de tiempo de diferentes de registros históricos y de los puntos de rejilla más cercanos a la isla de distintas bases de datos geofísicas públicas. Se hizo énfasis en la recolección de registros de precipitación, viento, radiación, humedad relativa, temperatura superficial del aire y del mar. Para todas las variables con registros de datos horarios, se obtuvieron sus ciclos diarios y anuales y se estudió sus características intra-estacionales, tales como máximos, mínimos y épocas de transición; y se hizo la comparación de los registros de precipitación de las estaciones con los registros de las bases de datos con mayor escala espacial. Se observa que las variaciones estacionales en la Isla del Coco están asociadas a la migración meridional de la Zona de Convergencia Inter-Tropical, ya que la isla se encuentra bajo su influencia prácticamente desde la primavera hasta el otoño boreal.

Palabras clave: Isla del Coco, Pacífico Tropical Este, climatología, variabilidad intra-estacional, Zona de Convergencia Inter-Tropical.

\section{REFERENCIAS}

Alfaro, E. \& J. A. Amador. 1997. Variabilidad y cambio climático en algunos parámetros sobre Costa Rica y su relación con fenómenos de escala sinóptica y planetaria. Tóp. Meteorol. Oceanogr. 4: 51-62.

Amador, J., E. Alfaro, O. Lizano \& V. Magaña. 2006. Atmospheric forcing in the Eastern Tropical Pacific: A review. Progr. Oceanogr. 69: 101-142.

Ballance, L.T., R.L. Pitman \& P.C. Fiedler. 2006. Seabirds, cetaceans and the oceanography of the eastern tropical Pacific: a review. Progr. Oceanogr. 69: 360-390.

Chelton, D.B., M.G. Schlax, M.H. Freilich, \& R.F Milliff. 2004. Satellite measurements reveal persistent smallscale features in ocean winds. Science 303: 978-983.

Cortés, J. 2008. Historia de la investigación marina de la Isla del Coco, Costa Rica. Rev. Biol. Trop. 56 (Supl. 2): 1-18.

da Silva, A., A.C. Young, \& S. Levitus. 1994. Atlas of Surface Marine Data, vol. 1: Algorithms and Procedures. NOAA Atlas NESDIS 6, US Department of Commerce, Washington, D.C.

Douglas, M.W., W. Fernández \& M. Peña. 1999. Design and evolution of the PACS SONET observing system in Latin America, p. 131-134. Third Symp. Integrated Observing Systems, Dallas, Texas, 10-15 January 1999.

Fernández-Álamo, M.A. \& J. Färber-Lorda. 2006. Zooplankton and the oceanography of the eastern tropical Pacific: A review. Progr. Oceanogr. 69: 318-359.

Fernández, W. 1984. Comments on meteorological and climatological observations on Coco Island. Rev. Geofís. 20: 9-19.

Fiedler, P. \& L. Talley. 2006. Hydrography of the eastern tropical Pacific: A review. Progr. Oceanogr. 69: 143-180.

IPCC, 2007: Summary for Policymakers. In: Climate Change 2007: The Physical Science Basis. Contribution of Working Group I to the Fourth Assessment Report of the Intergovernmental Panel on Climate Change [Solomon, S., D. Qin, M. Manning, Z. Chen, M. Marquis, K.B. Averyt, M.Tignor and H.L. Miller (eds.)]. Cambridge University Press, Cambridge, United Kingdom and New York, NY, USA. 
Kaplan A., M.A. Cane, Y. Kushnir, A.C. Clement, M.B. Blumenthal \& B. Rajagopalan. 1998. Analysis of global sea surface temperatures 1856-1991. J. Geophys. Res. 103: 18567-18589.

Kessler, W.S. 2006. The circulation of the eastern tropical Pacific: A review. Progr. Oceanogr. 69: 181-217.

Lizano, O. 2001. Batimetría de la plataforma insular alrededor de la Isla del Coco, Costa Rica, Centro América. Rev. Biol. Trop. 49 (Supl. 2): 163-170.

Lizano, O. 2008. Dinámica de aguas alrededor de la Isla del Coco, Costa Rica. Rev. Biol. Trop. 56 (Supl. 2): 31-48.

Magaña, V.O., J.A. Amador \& S. Medina. 1999. The midsummer drought over Mexico and Central America. J. Climate 12: 1577-1588.

Morales-Ramírez, A. 2008. Caracterización cualitativa del zooplancton del Área de Conservación Marina Isla de Coco (ACMIC), Océano Pacífico de Costa Rica. Rev. Biol. Trop. 56 (Supl. 2): 159-169.

Muñoz, A., W. Fernández, J. Gutiérrez \& E. Zárate. 2002. Variación estacional del viento en Costa Rica y su relación con los regímenes de lluvia. Tóp. Meteorol. Oceanogr. 9: 1-13.

Pennington, J.T., K.L. Mahoney, V.S. Kuwahara, D.D. Kolber, R. Calienes \& F.P. Chavez. 2006. Primary productivity in the eastern tropical Pacific: A review. Progr. Oceanogr. 69: 285-317.

Philander, G. 1990. El Niño, La Niña and the Southern Oscillation. Academic, San Diego, California. 293 p.
Pittier, H.F. 1898. Apuntamientos preliminares sobre la Isla del Coco, posesión costarricense en el Océano Pacífico, p. 15-28. En Reproducciones Científicas, una Expedición y Legislación de la Isla del Coco. Instituto Geográfico de Costa Rica, Ministerio de Transportes. San José, Costa Rica, 1963.

Protti, E. 1964. Ensayo sobre el clima de la Isla del Coco, p. 27-28. En Nuevos apuntes y bibliografía de la Isla del Coco. Serie geográfica No. 2, Instituto Geográfico de Costa Rica, Ministerio de Transportes. San José, Costa Rica.

Reyes, S. \& G. Vogel. 1981. Meteorological and climatological observations on Coco Island. Rev. Geofís. 14/15: 85-93.

Rojas-Acuña, O. 1964. Informe general de las observaciones realizadas en la Isla del Coco, p. 21-26. En: Nuevos apuntes y bibliografía de la Isla del Coco. Serie geográfica No. 2, Instituto Geográfico de Costa Rica, Ministerio de Trasportes. San José, Costa Rica.

Willett, C.S., R. Leben \& M.F. Lavín. 2006. Eddies and mesoscale processes in the eastern tropical Pacific: A review. Progr. Oceanogr. 69: 218-238.

Xie, P. \& P. Arkin. 1996. Analyses of global monthly precipitation using gauge observations, satellite estimates, and numerical model predictions. J. Climate 9: 840-858.

Zhang, C., M. McGauley \& N.A. Bond. 2004. Shallow meridional circulation in the tropical eastern Pacific. J. Climate 17: 133-139. 


\section{APÉNDICE}

Una de las primeras descripciones del clima de la Isla del Coco fue publicada por Henri F. Pittier en 1898. Debido a su valor histórico, su acertada descripción y a que la fuente bibliográfica es difícil de conseguir fuera del ámbito costarricense, se reproduce a continuación el texto original de dicha descripción:

"El Clima.-Es naturalmente el océano típico. La temperatura media es de 25 grados centígrados, poco más ó menos, con poca fluctuación de una estación a otra, y reducida amplitud entre extremos. La atmósfera se conserva siempre muy húmeda y aunque hay indicios de una estación seca y de otra lluviosa, casi sería el caso de aplicar aquí el dicho de aquel Capitán inglés que afirmaba que la costa setentrional de Costa Rica demostraba dos estaciones anuales, (a wet season and a rainy season) una húmeda y la otra lluviosa. De junio á noviembre los vientos dominantes son del Suroeste, mientras soplan de entre Norte y Este en el resto del año. Sin embargo esta regla no tiene nada de fijo, por estar la isla en plena zona de calmas y de los vientos irregulares, y no dejaré de agregar que todas estas indicaciones son meras inducciones mías, apoyadas con los pocos datos que logré recoger en nuestra corta permanencia en la isla.

Importante para la climatología del Océano Pacífico y de mucho interés práctico para Costa Rica sería la organización de un pequeño Observatorio en la isla. El señor Gissler tendría sin duda alguna el mayor placer en hacerse cargo de él, y el Instituto Físico-Geográfico está en disposición para proporcionar los indispensables elementos.”

Nótese del último párrafo de esta cita, que ya desde finales del siglo XIX, se considera de vital importancia, a nivel costarricense y regional, las observaciones continuas de distintos parámetros geofísicos en la Isla del Coco, esto para el entendimiento del sistema climático en el Pacífico Tropical del Este. 
